\title{
Optimal harvesting control for an age-dependent competing population with diffusion
}

\author{
Jun $\mathrm{Fu}^{1 *}$, Xiulan $\mathrm{Wu}^{1}$ and Hong $\mathrm{Zhu}^{2}$
}

"Correspondence: jlfj8080@126.com 'Institute of Mathematics, Jilin Normal University, Siping, 136000, P.R. China

Full list of author information is available at the end of the article

\begin{abstract}
This paper is mainly concerned with optimal harvesting policy for competing species with age dependence and diffusion. The existence and uniqueness of solutions for the system are proved by using the Banach fixed point theorem. The existence and uniqueness of optimal control are discussed by Ekeland's variational principle. The maximum principle is obtained.
\end{abstract}

MSC: 35B10; 49K05; 65L12; 92B05

Keywords: competing; diffusion; optimal control; Ekeland's principle; maximum principle

\section{Introduction}

The optimal harvesting control of age-structured and size-structured single species have been widely studied in the literature [1-11]. The control problems of multi-species have been investigated in the literature [12-20]. The objective function represents the total harvesting, respectively, in [12-15]. The diffusion factor has not been considered in [1420]. It is well known that the profit due to harvesting is a quite important problem for optimal harvesting. In this paper, our purpose is to consider the optimal control of the profit functional for diffusion population. Specifically, we deal with the following optimal harvesting problem:

$$
\sup \sum_{i=1}^{n} \int_{Q}\left[u_{i}(a, t, x) p_{i}^{u}(a, t, x)-u_{i}^{2}(a, t, x)\right] \mathrm{d} a \mathrm{~d} t \mathrm{~d} x
$$

for all $u=\left(u_{1}(a, t, x), u_{2}(a, t, x), \ldots, u_{n}(a, t, x)\right) \in \mathcal{U}$, where the corresponding state variable $p^{u}=\left(p_{1}^{u}, p_{2}^{u}, \ldots, p_{n}^{u}\right)$ satisfies the state system

$$
\left\{\begin{aligned}
& \frac{\partial p_{i}}{\partial a}+\frac{\partial p_{i}}{\partial t}-k_{i} \Delta p_{i}= f_{i}-\mu_{i}(a, t, x) p_{i}-u_{i}(a, t, x) p_{i} \\
& \quad-\sum_{k=1, k \neq i}^{n} \lambda_{i k}(a, t, x) P_{k}(t, x) p_{i}, \quad(a, t, x) \in Q \\
& \frac{\partial p_{i}}{\partial v}(a, t, x)=0, \quad(a, t, x) \in \Sigma, \\
& p_{i}(0, t, x)=\int_{0}^{A} \beta_{i}(a, t, x) p_{i}(a, t, x) \mathrm{d} a, \quad(t, x) \in \Omega_{T}, \\
& p_{i}(a, 0, x)=p_{i 0}(a, x), \quad(a, x) \in \Omega_{A}, \\
& P_{i}(t, x)=\int_{0}^{A} p_{i}(a, t, x) \mathrm{d} a, \quad(t, x) \in \Omega_{T}, i=1,2, \ldots, n,
\end{aligned}\right.
$$


where $p_{i}(a, t, x)$ represents the density of the $i$ th population. $k_{i}$ is the diffusion rate of the $i$ th population. We assume that the populations have the same life expectancy $A$, $0<A<+\infty . \mu_{i}(a, t, x)$ is the average morality of the $i$ th population, and $\beta_{i}(a, t, x)$ describes the average fertility of the $i$ th population. $\lambda_{i k}(a, t, x)$ represents the interaction coefficients $(i, k=1,2, \ldots, n, k \neq i) . \Omega \subset R^{N}(N=1,2,3)$ is a bounded domain with a smooth enough boundary $\partial \Omega, Q=(0, A) \times(0, T) \times \Omega, \Omega_{T}=\Omega \times(0, T), \Omega_{A}=\Omega \times(0, A)$, $\sum=(0, A) \times(0, T) \times \partial \Omega$, and $v$ is the outward unit normal. The function $p_{i 0}(a, x)$ gives the initial density distribution of the population, and $u_{i}(a, t, x)$ represents the harvesting effort function, which is the control variable in the model and satisfies

$$
u_{i} \in \mathcal{U}_{i}=\left\{v_{i} \in L^{2}(Q) \mid 0 \leq \gamma_{i 1}(a, t, x) \leq v_{i}(a, t, x) \leq \gamma_{i 2}(a, t, x) \text { a.e. in } Q\right\}, \quad \mathcal{U}=\prod_{i=1}^{n} \mathcal{U}_{i},
$$

where $\gamma_{i j} \in L^{\infty}(Q), j=1,2, i=1,2, \ldots, n$. The integral

$$
J\left(u_{1}, u_{2}, \ldots, u_{n}\right)=\sum_{i=1}^{n} \int_{Q}\left[u_{i}(a, t, x) p_{i}^{u}(a, t, x)-u_{i}^{2}(a, t, x)\right] \mathrm{d} a \mathrm{~d} t \mathrm{~d} x
$$

represents the profit due to harvesting.

Throughout this paper, we assume that:

$\left(\mathrm{A}_{1}\right) \beta_{i}(a, t, x) \in L^{\infty}(Q), 0 \leq \beta_{i}(a, t, x) \leq M$, where $M$ is a constant.

$\left(\mathrm{A}_{2}\right) \mu_{i} \in L_{\mathrm{loc}}^{\infty}([0, A] \times[0, T] \times \bar{\Omega}), \mu_{i}(a, t, x) \geq \mu_{0}(a, t) \geq 0$ a.e. in $Q, i=1,2, \ldots, n$, where $\mu_{0} \in L_{\mathrm{loc}}^{\infty}([0, A] \times[0, T]), \int_{0}^{A} \mu_{0}(a, t+a-A) \mathrm{d} a=+\infty$.

$\left(\mathrm{A}_{3}\right) \lambda_{i k} \in L^{\infty}(Q), 0 \leq \lambda_{i k}(a, t, x) \leq B$, where $B$ is a positive constant $(i, k=1,2, \ldots, n, k \neq i)$.

$\left(\mathrm{A}_{4}\right) f_{i} \in L^{\infty}(Q), p_{i 0}(a, x) \in L^{\infty}\left(\Omega_{A}\right), p_{i 0}(a, x) \geq 0,0 \leq f_{i}(a, t, x) \leq B_{0}$, where $B_{0}$ is a constant, $i=1,2, \ldots, n$.

The rest of this paper is organized as follows. In Section 2, we prove that under the assumptions listed above, the system has a unique non-negative solution. In Section 3, the necessary conditions of optimality for the control problems is given. In the final section, we prove the existence and uniqueness of the optimal control.

\section{The existence and uniqueness of solution for system (1.2)}

For the sake of convenience, we introduce the following definitions of the solution.

Definition 2.1 Given the solution of system (1.2), the function $p_{i} \in L^{2}(Q), i=1,2, \ldots, n$, which belongs to $C\left(\bar{S} ; L^{2}(\Omega)\right) \cap A C\left(S ; L^{2}(\Omega)\right) \cap L^{2}\left(S ; H^{1}(\Omega)\right) \cap L_{\text {loc }}^{2}\left(S ; H^{2}(\Omega)\right)$ for almost any characteristic line $S$ of the equation

$$
a-t=a_{0}-t_{0}, \quad(a, t) \in(0, A) \times(0, T),\left(a_{0}, t_{0}\right) \in\{0\} \times(0, T) \cup(0, A) \times\{0\},
$$

satisfies

$$
\left\{\begin{aligned}
& \frac{\partial p_{i}}{\partial a}+\frac{\partial p_{i}}{\partial t}-k_{i} \Delta p_{i}= f_{i}-\mu_{i}(a, t, x) p_{i}-u_{i}(a, t, x) p_{i} \\
&-\sum_{k=1, k \neq i}^{n} \lambda_{i k}(a, t, x) P_{k}(t, x) p_{i}, \quad \text { a.e. in } Q, \\
& \frac{\partial p_{i}}{\partial v}(a, t, x)=0, \quad \text { a.e. in } \Sigma, \\
& \lim _{\varepsilon \rightarrow 0^{+}} p(\varepsilon, t+\varepsilon, \cdot)=\int_{0}^{A} \beta_{i}(a, t, \cdot) p_{i}(a, t, \cdot) \mathrm{d} a, \quad \text { in } L^{2}(\Omega), \text { a.e. } t \in(0, T), \\
& \lim _{\varepsilon \rightarrow 0^{+}} p_{i}(a+\varepsilon, \varepsilon, \cdot)=p_{i 0}(a, \cdot), \quad \text { in } L^{2}(\Omega), \text { a.e. } a \in(0, A),
\end{aligned}\right.
$$


where $P_{i}(t, \cdot)=\int_{0}^{A} p_{i}(a, t, \cdot) \mathrm{d} a, i=1,2, \ldots, n$.

Then we rewrite the characteristic line $S$ as

$$
S=\left\{(a, t) \in(0, A) \times(0, T) ; a-t=a_{0}-t_{0}\right\}=\left\{\left(a_{0}+s, t_{0}+s\right) ; s \in(0, \alpha)\right\}
$$

here $\left(a_{0}+\alpha, t_{0}+\alpha\right) \in\{A\} \times(0, T) \cup(0, A) \times\{T\}$.

We introduce the following notations:

$$
\begin{aligned}
& C\left(\bar{S} ; L^{2}(\Omega)\right)=\left\{h: \bar{S} \rightarrow L^{2}(\Omega) ; h \text { continuous }\right\} \\
& A C\left(S ; L^{2}(\Omega)\right)=\left\{h: S \rightarrow L^{2}(\Omega): h\left(a_{0}+\cdot, t_{0}+\cdot\right):(0, \alpha) \rightarrow L^{2}(\Omega)\right.
\end{aligned}
$$

is absolutely continuous on any compact subinterval\}.

Theorem 2.1 For any given $u=\left(u_{1}, u_{2}, \ldots, u_{n}\right) \in \mathcal{U}$, system (1.2) has a unique non-negative solution $p^{u}=\left(p_{1}^{u}, p_{2}^{u}, \ldots, p_{n}^{u}\right) \in L^{2}\left(Q ; R^{n}\right)$ such that

(i) $0 \leq p_{i}^{u} \leq M_{1}, \forall(a, t, x) \in Q, i=1,2, \ldots, n$, where $M_{1}$ is a positive constant;

(ii) $p^{u}$ is continuous in $u$.

Proof For any given $h=\left(h_{1}, h_{2}, \ldots, h_{n}\right) \in L^{2}\left(Q ; R^{n}\right), h \geq 0$, we define

$$
H_{i}(t, x)=\int_{0}^{A} h_{i}(a, t, x) \mathrm{d} a, \quad i=1,2, \ldots, n .
$$

The given system

$$
\left\{\begin{array}{l}
\frac{\partial p_{i}}{\partial a}+\frac{\partial p_{i}}{\partial t}-k_{i} \Delta p_{i}=f_{i}-\mu_{i}(a, t, x) p_{i}-u_{i} p_{i} \\
\quad-\sum_{k=1, k \neq i}^{n} \lambda_{i k}(a, t, x) H_{k}(t, x) p_{i}, \quad(a, t, x) \in Q \\
\frac{\partial p_{i}}{\partial v}(a, t, x)=0, \quad(a, t, x) \in \Sigma, \\
p_{i}(0, t, x)=\int_{0}^{A} \beta_{i}(a, t, x) p_{i}(a, t, x) \mathrm{d} a, \quad(t, x) \in \Omega_{T}, \\
p_{i}(a, 0, x)=p_{i 0}(a, x), \quad(a, x) \in \Omega_{A}, \\
P_{i}(t, x)=\int_{0}^{A} p_{i}(a, t, x) \mathrm{d} a, \quad(t, x) \in \Omega_{T}, i=1,2, \ldots, n,
\end{array}\right.
$$

by Theorem 4.1.3 in [21], we know that the above system has a unique non-negative solution,

$$
p^{h}=\left(p_{1}^{h}, p_{2}^{h}, \ldots, p_{n}^{h}\right) \in L^{2}\left(Q ; R^{n}\right), \quad p_{i}^{h}(A, t, x)=0, \quad \forall(t, x) \in \Omega_{T}, i=1,2, \ldots, n .
$$

From the comparison principle of linear system [21], it follows that

$$
p_{i}^{h}(a, t, x) \leq \bar{p}_{i}(a, t, x), \quad \text { a.e. in } Q, i=1,2, \ldots, n,
$$

where $\bar{p}_{i}(a, t, x) \in L^{\infty}(Q)$, and this is the solution of the following system:

$$
\left\{\begin{array}{l}
\frac{\partial p_{i}}{\partial a}+\frac{\partial p_{i}}{\partial t}-k_{i} \Delta p_{i}=f_{i}-\mu_{i}(a, t, x) p_{i}, \quad(a, t, x) \in Q, \\
\frac{\partial p_{i}}{\partial v}(a, t, x)=0, \quad(a, t, x) \in \Sigma, \\
p_{i}(0, t, x)=\int_{0}^{A} \beta_{i}(a, t, x) p_{i}(a, t, x) \mathrm{d} a, \quad(t, x) \in \Omega_{T}, \\
p_{i}(a, 0, x)=p_{i 0}(a, x), \quad(a, x) \in \Omega_{A} .
\end{array}\right.
$$


For any $h^{k}=\left(h_{1}^{k}, h_{2}^{k}, \ldots, h_{n}^{k}\right) \in L^{2}\left(Q ; R^{n}\right), 0 \leq h_{i}^{k} \leq \bar{p}_{i}$, let the corresponding state be $p^{k}=$ $\left(p_{1}^{k}, p_{2}^{k}, \ldots, p_{n}^{k}\right)(k=1,2), w=\left(w_{1}, w_{2}, \ldots, w_{n}\right):=p^{1}-p^{2}$. It follows from (1.2) that

$$
\left\{\begin{array}{l}
\frac{\partial w_{i}}{\partial a}+\frac{\partial w_{i}}{\partial t}-k_{i} \Delta w_{i}=-\left(\mu_{i}+u_{i}\right) w_{i}-\sum_{k=1, k \neq i}^{n} \lambda_{i k}(a, t, x) H_{k}^{1}(t, x) w_{i} \\
\quad-\sum_{k=1, k \neq i}^{n} \lambda_{i k}(a, t, x)\left[H_{k}^{1}(t, x)-H_{k}^{2}(t, x)\right] p_{i}^{2}, \quad(a, t, x) \in Q \\
\frac{\partial w_{i}}{\partial v}(a, t, x)=0, \quad(a, t, x) \in \Sigma, \\
w_{i}(0, t, x)=\int_{0}^{A} \beta_{i}(a, t, x) w_{i}(a, t, x) \mathrm{d} a \\
w_{i}(a, 0, x)=0, \quad(a, x) \in \Omega_{A}, \\
H_{i}^{j}(t, x)=\int_{0}^{A} h_{i}^{j}(a, t, x) \mathrm{d} a, \quad(t, x) \in \Omega_{T}, i=1,2, \ldots, n, j=1,2 .
\end{array}\right.
$$

Multiplying the $i$ th equation in system $(2.3)$ by $w_{i}(i=1,2, \ldots, n)$, integrating on $(0, A) \times$ $(0, t) \times \Omega$, and according to the Hölder inequality, we have

$$
\begin{aligned}
\left\|w_{i}(\cdot, t, \cdot)\right\|_{L^{2}\left(\Omega_{A}\right)}^{2} & \\
\leq & \left(A M^{2}+B\left\|\bar{p}_{i}\right\|_{L^{\infty}(Q)}\right) \int_{0}^{t}\left\|w_{i}(\cdot, \tau, \cdot)\right\|_{L^{2}\left(\Omega_{A}\right)}^{2} \mathrm{~d} \tau \\
& +\sum_{k=1}^{n} A^{2} B\left\|\bar{p}_{i}\right\|_{L^{\infty}(Q)} \int_{0}^{t}\left\|h_{k}^{1}-h_{k}^{2}\right\|_{L^{2}\left(\Omega_{A}\right)}^{2} \mathrm{~d} \tau \\
=\widetilde{C} & \int_{0}^{t}\left(\sum_{k=1}^{n}\left\|h_{k}^{1}(\cdot, \tau, \cdot)-h_{k}^{2}(\cdot, \tau, \cdot)\right\|_{L^{2}\left(\Omega_{A}\right)}^{2}+\left\|w_{i}(\cdot, \tau, \cdot)\right\|_{L^{2}\left(\Omega_{A}\right)}^{2}\right) \mathrm{d} \tau
\end{aligned}
$$

where $\widetilde{C}=\max _{1 \leq i \leq n}\left\{A M^{2}+B\left\|\bar{p}_{i}\right\|_{L^{\infty}(Q)}, A^{2} B\left\|\bar{p}_{i}\right\|_{L^{\infty}(Q)}\right\}$. By using the Gronwall inequality, we have

$$
\left\|w_{i}(\cdot, t, \cdot)\right\|_{L^{2}\left(\Omega_{A}\right)}^{2} \leq C \sum_{k=1}^{n} \int_{0}^{t}\left\|h_{k}^{1}(\cdot, \tau, \cdot)-h_{k}^{2}(\cdot, \tau, \cdot)\right\|_{L^{2}\left(\Omega_{A}\right)}^{2} \mathrm{~d} \tau
$$

where $C=\widetilde{C} e^{\widetilde{C} T}$. Set

$$
I:=\left\{h=\left(h_{1}, h_{2}, \ldots, h_{n}\right) \in L^{2}\left(Q ; R^{n}\right): 0 \leq h_{i}(a, t, x) \leq \bar{p}_{i}(a, t, x), \forall(a, t, x) \in Q\right\} .
$$

Define the mapping $G: I \rightarrow I$

$$
(G h)(a, t, x)=p^{h}(a, t, x), \quad \forall(a, t, x) \in Q
$$

Denote the norm on $L^{2}\left(Q ; R^{N}\right)$ by

$$
\|h\|_{L^{2}(Q)}=\left(\sum_{i=1}^{n}\left\|h_{i}\right\|_{L^{2}(Q)}^{2}\right)^{\frac{1}{2}}
$$

Define the following norm for $C>0$ :

$$
\left\|h_{i}\right\|_{*}=\int_{0}^{T}\left\|h_{i}(\cdot, t)\right\|_{L^{2}\left(\Omega_{A}\right)}^{2} e^{-4 n C t} \mathrm{~d} t, \quad\|h\|_{*}^{2}=\left(\sum_{i=1}^{n}\left\|h_{i}\right\|_{*}^{2}\right)^{\frac{1}{2}}, \quad i=1,2, \ldots, n
$$


Obviously the norm $\|\cdot\|_{L^{2}(Q)}$ is equivalent to the norm $\|\cdot\|_{*}$. Using (2.5), we get

$$
\begin{aligned}
\left\|G h^{1}-G h^{2}\right\|_{*} & =\left\|p^{1}-p^{2}\right\|_{*} \\
& =\left(\sum_{i=1}^{n}\left\|w_{i}\right\|_{*}^{2}\right)^{\frac{1}{2}} \\
& =\left(\sum_{i=1}^{n} \int_{0}^{T}\left\|w_{i}(\cdot, t, \cdot)\right\|_{L^{2}\left(\Omega_{A}\right)}^{2} e^{-4 n C t} \mathrm{~d} t\right)^{\frac{1}{2}} \\
& \leq\left(\sum_{i=1}^{n} \int_{0}^{T} C \sum_{k=1}^{n} \int_{0}^{t}\left\|h_{k}^{1}(\cdot, \tau, \cdot)-h_{k}^{2}(\cdot, \tau, \cdot)\right\|_{L^{2}\left(\Omega_{A}\right)}^{2} \mathrm{~d} \tau \cdot e^{-4 n C t} \mathrm{~d} t\right)^{\frac{1}{2}} \\
& =\left(\int_{0}^{T} \sum_{k=1}^{n}\left\|h_{k}^{1}(\cdot, s, \cdot)-h_{k}^{2}(\cdot, s, \cdot)\right\|_{L^{2}\left(\Omega_{A}\right)}^{2} \cdot \int_{s}^{T} n C e^{-4 n C t} \mathrm{~d} t \mathrm{~d} s\right)^{\frac{1}{2}} \\
& \leq \frac{1}{2}\left(\int_{0}^{T} \sum_{k=1}^{n}\left\|h_{k}^{1}(\cdot, s, \cdot)-h_{k}^{2}(\cdot, s, \cdot)\right\|_{L^{2}\left(\Omega_{A}\right)}^{2} \cdot e^{-4 n C s} \mathrm{~d} s\right)^{\frac{1}{2}} \\
& =\frac{1}{2}\left\|h_{1}-h_{2}\right\|_{*}
\end{aligned}
$$

It is obvious that $G I \subseteq I$ and $G$ is a contraction on $\left(I,\|\cdot\|_{*}\right)$, so there is a unique fixed point, which is the solution of system (1.2).

Since $\bar{p}_{i} \in L^{\infty}(Q)$, let $M_{1}=\max \left\{\operatorname{ess} \sup \left|\bar{p}_{i}(a, t, x)\right|, 1 \leq i \leq n\right\}$, we have $0 \leq p_{i}^{u} \leq M_{1}$, a.e. $(a, t, x) \in Q$. In the following, we study the continuity of the solution of (1.2) for the control variable $u$. Let $u^{k}=\left(u_{1}^{k}, u_{2}^{k}, \ldots, u_{n}^{k}\right) \in \mathcal{U}, k=1,2, y_{i}(a, t, x)=p_{i}^{u^{1}}(a, t, x)-p_{i}^{u^{2}}(a, t, x)$. From (1.2), it follows that

$$
\left\{\begin{aligned}
& \frac{\partial y_{i}}{\partial a}+\frac{\partial y_{i}}{\partial t}-k_{i} \Delta y_{i}=-\left(\mu_{i}+u_{i}^{1}\right) y_{i} \\
&-\sum_{k=1, k \neq i}^{n} \lambda_{i k} p_{i}^{u^{2}} \int_{0}^{A} y_{k}(a, t, x) \mathrm{d} a-\left(u_{i}^{1}-u_{i}^{2}\right) p_{i}^{u^{2}} \\
&-\sum_{k=1, k \neq i}^{n} \lambda_{i k}(a, t, x) P_{k}^{u^{1}}(t, x) y_{i}, \quad(a, t, x) \in Q, \\
& \frac{\partial y_{i}}{\partial v}(a, t, x)=0, \quad(a, t, x) \in \Sigma, \\
& y_{i}(0, t, x)=\int_{0}^{A} \beta_{i}(a, t, x) y_{i}(a, t, x) \mathrm{d} a, \quad(t, x) \in \Omega_{T}, \\
& y_{i}(a, 0, x)=0, \quad(a, x) \in \Omega_{A}, \\
& P_{i}^{u^{j}}(t, x)=\int_{0}^{A} p_{i}^{u^{j}}(a, t, x) \mathrm{d} a, \quad(t, x) \in \Omega_{T}, i=1,2, \ldots, n, j=1,2 .
\end{aligned}\right.
$$

In a similar manner as that in (2.4), we deduce that

$$
\begin{aligned}
\left\|y_{i}(\cdot, \tau, \cdot)\right\|_{L^{2}\left(\Omega_{A}\right)}^{2} \leq & \left(A M^{2}+\left\|\bar{p}_{i}\right\|_{L^{\infty}(Q)}+B\left\|\bar{p}_{i}\right\|_{L^{\infty}(Q)}\right) \int_{0}^{t}\left\|y_{i}(\cdot, \tau, \cdot)\right\|_{L^{2}\left(\Omega_{A}\right)}^{2} \mathrm{~d} \tau \\
& +\left\|\bar{p}_{i}\right\|_{L^{\infty}(Q)} \int_{0}^{t}\left\|u_{i}^{1}-u_{i}^{2}\right\|_{L^{2}\left(\Omega_{A}\right)}^{2} \mathrm{~d} \tau \\
& +2 \sum_{k=1, k \neq i}^{n} A B\left\|\bar{p}_{k}\right\|_{L^{\infty}(Q)} \int_{0}^{t} \int_{\Omega_{A}} y_{i}^{2} \mathrm{~d} x \mathrm{~d} a \mathrm{~d} \tau .
\end{aligned}
$$


Let $\bar{C}=A M^{2}+\left\|\bar{p}_{i}\right\|_{L^{\infty}(Q)}+B\left\|\bar{p}_{i}\right\|_{L^{\infty}(Q)}+2 A B \sum_{k=1, k \neq i}^{n}\left\|\bar{p}_{k}\right\|_{L^{\infty}(Q)}$, we have

$$
\begin{aligned}
\left\|y_{i}(\cdot, t, \cdot)\right\|_{L^{2}\left(\Omega_{A}\right)}^{2} \leq & \bar{C}\left(\int_{0}^{t}\left\|u_{i}^{1}(\cdot, \tau, \cdot)-u_{i}^{2}(\cdot, \tau, \cdot)\right\|_{L^{2}\left(\Omega_{A}\right)}^{2} \mathrm{~d} \tau\right. \\
& \left.+\int_{0}^{t}\left\|y_{i}(\cdot, \tau, \cdot)\right\|_{L^{2}\left(\Omega_{A}\right)}^{2} \mathrm{~d} \tau\right) .
\end{aligned}
$$

Adding up (2.7) from $i=1$ to $n$ and using the Gronwall inequality, we get

$$
\sum_{i=1}^{n}\left\|y_{i}(\cdot, t, \cdot)\right\|_{L^{2}\left(\Omega_{A}\right)}^{2} \leq K \sum_{i=1}^{n} \int_{0}^{t}\left\|u_{i}^{1}(\cdot, \tau, \cdot)-u_{i}^{2}(\cdot, \tau, \cdot)\right\|_{L^{2}\left(\Omega_{A}\right)}^{2} \mathrm{~d} \tau
$$

where $K=\bar{C} e^{\bar{C} T}$. Multiplying (2.8) by $e^{-n r t}$ and integrating on $(0, T)$, we have

$$
\left\|p^{1}-p^{2}\right\|_{*} \leq \sqrt{K T}\left\|u^{1}-u^{2}\right\|_{*}
$$

Inequality (2.9) implies that $p^{u}$ is continuous with respect to $u$. This completes the proof.

\section{The necessary conditions}

Before stating our main results, we prove the following lemma, which is useful in proving our results.

Lemma 3.1 Let $\left(u^{*}, p^{*}\right)$ be an optimal pair for Problem (1.1), for any $0<\varepsilon<1$ and for any $v=\left(v_{1}, v_{2}, \ldots, v_{n}\right) \in L^{2}\left(Q ; R^{n}\right), v_{i}>0, u^{*}+\varepsilon\left(v-u^{*}\right)=u_{\varepsilon} \in \mathcal{U}$, where $u_{\varepsilon}=\left(u_{1 \varepsilon}, u_{2 \varepsilon}, \ldots, u_{n \varepsilon}\right)$, then the following limit holds:

$$
\frac{p^{u_{\varepsilon}}-p^{u^{*}}}{\varepsilon} \rightarrow z, \quad \text { in } L^{2}\left(Q ; R^{n}\right) \text {, as } \varepsilon \rightarrow 0^{+}
$$

where $p^{*}=\left(p_{1}^{u^{*}}, p_{2}^{u^{*}}, \ldots, p_{n}^{u^{*}}\right), u^{*}=\left(u_{1}^{*}, u_{2}^{*}, \ldots, u_{n}^{*}\right)$, and $z=\left(z_{1}, z_{2}, \ldots, z_{n}\right)$ is the solution of

$$
\left\{\begin{aligned}
& \frac{\partial z_{i}}{\partial a}+\frac{\partial z_{i}}{\partial t}-k_{i} \Delta z_{i}=-\left(\mu_{i}(a, t, x)+u_{i}^{*}\right) z_{i}-\left(v_{i}-u_{i}^{*}\right) p_{i}^{u^{*}} \\
&-\sum_{k=1, k \neq i}^{n} \lambda_{i k}(a, t, x) Z_{k}(t, x) p_{i}^{u^{*}} \\
&-\sum_{k=1, k \neq i}^{n} \lambda_{i k} P_{k}^{u^{*}}(t, x) z_{i}, \quad(a, t, x) \in Q \\
& \frac{\partial z_{i}}{\partial v}(a, t, x)=0, \quad(a, t, x) \in \Sigma, \\
& z_{i}(0, t, x)=\int_{0}^{A} \beta_{i}(a, t, x) z_{i}(a, t, x) \mathrm{d} a, \quad(t, x) \in \Omega_{T}, \\
& z_{i}(a, 0, x)=0, \quad(a, x) \in \Omega_{A}, \\
& P_{i}^{u^{*}}(t, x)=\int_{0}^{A} p_{i}^{u^{*}}(a, t, x) \mathrm{d} a, \quad(t, x) \in \Omega_{T}, \\
& Z_{i}(t, x)=\int_{0}^{A} z_{i}(a, t, x) \mathrm{d} a, \quad(t, x) \in \Omega_{T}, i=1,2, \ldots, n .
\end{aligned}\right.
$$

Proof The existence and uniqueness of the solution of (3.1) follow in the same manner as by Theorem 2.1 , the solution $z=\left(z_{1}, z_{2}, \ldots, z_{n}\right) \in L^{2}\left(Q ; R^{n}\right)$, and $0 \leq z_{i} \leq L$, where $L$ is a constant. We introduce the following notations:

$$
\begin{aligned}
& z_{\varepsilon}(a, t, x)=\frac{1}{\varepsilon}\left[p^{u_{\varepsilon}}(a, t, x)-p^{u^{*}}(a, t, x)\right], \quad(a, t, x) \in Q, \\
& x_{\varepsilon}(a, t, x)=\varepsilon z_{\varepsilon}(a, t, x), \quad(a, t, x) \in Q .
\end{aligned}
$$


It is straightforward that $z_{\varepsilon}$ is the solution of

$$
\left\{\begin{aligned}
& \frac{\partial z_{i \varepsilon}}{\partial a}+\frac{\partial z_{i \varepsilon}}{\partial t}-k_{i} \Delta z_{i \varepsilon}=-\left(\mu_{i}+u_{i}^{*}\right) z_{i \varepsilon}-\left(v_{i}-u_{i}^{*}\right) p_{i}^{u_{\varepsilon}} \\
&-\sum_{k=1, k \neq i}^{n} \lambda_{i k}(a, t, x) Z_{k \varepsilon}(t, x) p_{i}^{u_{\varepsilon}} \\
&-\sum_{k=1, k \neq i}^{n} \lambda_{i k}(a, t, x) P_{k}^{u^{*}}(t, x) z_{i \varepsilon}, \quad(a, t, x) \in Q \\
& \frac{\partial z_{i \varepsilon}}{\partial v}(a, t, x)=0, \quad(a, t, x) \in \Sigma, \\
& z_{i \varepsilon}(0, t, x)=\int_{0}^{A} \beta_{i}(a, t, x) z_{i \varepsilon}(a, t, x) \mathrm{d} a, \quad(t, x) \in \Omega_{T}, \\
& z_{i}(a, 0, x)=0, \quad(a, x) \in \Omega_{A},
\end{aligned}\right.
$$

where $P_{i}^{u^{*}}(t, x)=\int_{0}^{A} p_{i}^{u^{*}}(a, t, x) \mathrm{d} a, Z_{i \varepsilon}(t, x)=\int_{0}^{A} z_{i \varepsilon}(a, t, x) \mathrm{d} a,(t, x) \in \Omega_{T}$.

By (2.9), we have

$$
\begin{aligned}
\left\|x_{\varepsilon}\right\|_{*} & =\left\|p^{u_{\varepsilon}}-p^{u^{*}}\right\|_{*} \\
& \leq \sqrt{K T}\left\|\varepsilon\left(v-u^{*}\right)\right\|_{*} \\
& =\sqrt{K T} \varepsilon\left\|v-u^{*}\right\|_{*} .
\end{aligned}
$$

So we can claim that $x_{\varepsilon} \rightarrow 0$ in $L^{2}\left(Q ; R^{n}\right)$, as $\varepsilon \rightarrow 0^{+}$. By (3.1) and (3.2), we see that $z_{\varepsilon}-z=\left(z_{1 \varepsilon}-z_{1}, z_{2 \varepsilon}-z_{2}, \ldots, z_{n \varepsilon}-z_{n}\right) \in L^{2}\left(Q ; R^{n}\right)$ is the solution of the following equation:

$$
\left\{\begin{array}{l}
\frac{\partial\left(z_{i \varepsilon}-z_{i}\right)}{\partial a}+\frac{\partial\left(z_{i \varepsilon}-z_{i}\right)}{\partial t}-k_{i} \Delta\left(z_{i \varepsilon}-z_{i}\right) \\
=-\mu_{i}\left(z_{i \varepsilon}-z_{i}\right)-u_{i}^{*}\left(z_{i \varepsilon}-z_{i}\right)-\left(v_{i}-u_{i}^{*}\right)\left(p_{i}^{u_{\varepsilon}}-p_{i}^{u^{*}}\right) \\
\quad-\sum_{k=1, k \neq i}^{n} \lambda_{i k}\left(\int_{0}^{A}\left(z_{k \varepsilon}-z_{k}\right) \mathrm{d} a\right) p_{i}^{u_{\varepsilon}} \\
\quad-\sum_{k=1, k \neq i}^{n} \lambda_{i k}(a, t, x) Z_{k}(t, x)\left(p_{i}^{u_{\varepsilon}}-p_{i}^{u^{*}}\right) \\
\quad+\sum_{k=1, k \neq i}^{n} \lambda_{i k}\left(\int_{0}^{A}\left(p_{k}^{u_{\varepsilon}}-p_{k}^{u^{*}}\right) \mathrm{d} a\right) z_{i \varepsilon} \\
\quad-\sum_{k=1, k \neq i}^{n} \lambda_{i k}\left(\int_{0}^{A} p_{k}^{u^{*}}(a, t, x) \mathrm{d} a\right)\left(z_{i \varepsilon}-z_{i}\right), \quad(a, t, x) \in Q, \\
\frac{\partial\left(z_{i \varepsilon}-z_{i}\right)}{\partial v}(a, t, x)=0, \quad(a, t, x) \in \Sigma, \\
\left(z_{i \varepsilon}-z_{i}\right)(0, t, x)=\int_{0}^{A} \beta_{i}(a, t, x)\left(z_{i \varepsilon}-z_{i}\right) \mathrm{d} a, \quad(t, x) \in \Omega_{T}, \\
z_{i}(a, 0, x)=0, \quad(a, x) \in \Omega_{A}, i=1,2, \ldots, n,
\end{array}\right.
$$

where $P_{i}^{u^{*}}(t, x)=\int_{0}^{A} p_{i}^{u^{*}}(a, t, x) \mathrm{d} a, Z_{i}(t, x)=\int_{0}^{A} z_{i}(a, t, x) \mathrm{d} a,(t, x) \in \Omega_{T}$.

Multiplying the first equation in (3.4) by $z_{i \varepsilon}-z_{i}$, integrating on $(0, A) \times(0, t) \times \Omega$, using the Gronwall inequality, in the same manner as in (2.5), and taking $x_{\varepsilon} \rightarrow 0$ in $L^{2}\left(Q ; R^{n}\right)$, we deduce

$$
z_{\varepsilon} \rightarrow z, \quad \text { in } L^{2}\left(Q, R^{n}\right) \text {, as } \varepsilon \rightarrow 0^{+}
$$

Consequently, the proof is completed. By the same argument as in (2.9), the following lemma holds.

In order to give the necessary conditions, we introduce the adjoint equations of (3.1). 
Lemma 3.2 If $q^{u^{1}}, q^{u^{2}}$ are the solutions of the following system corresponding to the control $u^{1}, u^{2}$, respectively:

$$
\left\{\begin{aligned}
& \frac{\partial q_{j}}{\partial a}+\frac{\partial q_{j}}{\partial t}+k_{j} \Delta q_{j}=\left(\mu_{j}+u_{j}^{*}\right) q_{j}+u_{j}^{*}-q_{j}(0, t, x) \beta_{j}(a, t, x) \\
&+\sum_{k=1, k \neq j}^{n} \lambda_{j k}(a, t, x) q_{j} P_{k}^{u^{*}}(t, x) \\
&+\sum_{k=1, k \neq j}^{n} \int_{0}^{A} \lambda_{k j}(a, t, x) p_{k}^{u^{*}} q_{j}(a, t, x) \mathrm{d} a, \quad(a, t, x) \in Q \\
& \frac{\partial q_{j}}{\partial \nu}(a, t, x)=0, \quad(a, t, x) \in \Sigma, \\
& q_{j}(a, T, x)=q_{j}(A, t, x)=0, \quad(a, x) \in \Omega_{A}, \\
& P_{j}^{u^{*}}(t, x)=\int_{0}^{A} p_{j}^{u^{*}}(a, t, x) \mathrm{d} a, \quad(t, x) \in \Omega_{T}, j=1,2, \ldots, n .
\end{aligned}\right.
$$

Then

$$
\begin{aligned}
& \left\|q^{u^{1}}-q^{u^{2}}\right\|_{*} \leq \sqrt{K T}\left\|u^{1}-u^{2}\right\|_{*^{\prime}} \\
& \left\|q^{u}\right\|_{*} \leq M_{2},
\end{aligned}
$$

where $q^{u^{1}}=\left(q_{1}^{u^{1}}, q_{2}^{u^{1}}, \ldots, q_{n}^{u^{1}}\right), q^{u^{2}}=\left(q_{1}^{u^{2}}, q_{2}^{u^{2}}, \ldots, q_{n}^{u^{2}}\right), u^{1}=\left(u_{1}^{1}, u_{2}^{1}, \ldots, u_{n}^{1}\right), u^{2}=\left(u_{1}^{2}, u_{2}^{2}\right.$, $\left.\ldots, u_{n}^{2}\right), \bar{K}>0, M_{2}$ are constant and independent of $q^{u^{1}}, q^{u^{2}}, u^{1}, u^{2}$.

We are now able to state the main result in this section.

Theorem 3.1 Suppose that $\left(\mathrm{A}_{1}\right)-\left(\mathrm{A}_{5}\right)$ hold, if $\left(u^{*}, p^{*}\right)$ is an optimal pair for Problem (1.1), and $q=\left(q_{1}, q_{2}, \ldots, q_{n}\right)$ is the solution of (3.5), which is corresponding to the control $u^{*}=$ $\left(u_{1}^{*}, u_{2}^{*}, \ldots, u_{n}^{*}\right)$, then we have the following necessary conditions:

$$
\sum_{i=1}^{n} \int_{0}^{A} \int_{0}^{T} \int_{\Omega}\left(v_{i}-u_{i}^{*}\right)\left[\left(1+q_{i}\right) p_{i}^{u^{*}}-2 u_{i}^{*}\right] \mathrm{d} x \mathrm{~d} t \mathrm{~d} a \leq 0 .
$$

Proof The existence and uniqueness of the solution $q=\left(q_{1}, q_{2}, \ldots, q_{n}\right)$ to (3.5) can be proved by Theorem 2.1. For any $v_{i} \in L^{2}(Q)$ and any $0<\varepsilon<1, u_{i}^{*}+\varepsilon\left(v_{i}-u_{i}^{*}\right) \in \mathcal{U}_{i}$. Since $u^{*}=\left(u_{1}^{*}, u_{2}^{*}, \ldots, u_{n}^{*}\right)$ is an optimal control for (1.1), we get

$$
\sum_{i=1}^{n} \int_{Q}\left[u_{i}^{*} p_{i}^{u^{*}}-\left(u_{i}^{*}\right)^{2}\right] \mathrm{d} a \mathrm{~d} t \mathrm{~d} x \geq \sum_{i=1}^{n} \int_{Q}\left[u_{i \varepsilon} p_{i}^{u_{\varepsilon}}-u_{i \varepsilon}^{2}\right] \mathrm{d} a \mathrm{~d} t \mathrm{~d} x
$$

which implies

$$
\sum_{i=1}^{n} \int_{0}^{A} \int_{0}^{T} \int_{\Omega}\left[u_{i}^{*} \frac{p_{i}^{u_{\varepsilon}}-p_{i}^{u^{*}}}{\varepsilon}+\left(v_{i}-u_{i}^{*}\right)\left(p_{i}^{u_{\varepsilon}}-2 u_{i}^{*}-\varepsilon\left(v_{i}-u_{i}^{*}\right)\right)\right] \mathrm{d} x \mathrm{~d} t \mathrm{~d} a \leq 0
$$

By Lemma 3.1 and inequality (3.3), passing to the limit as $\varepsilon \rightarrow 0^{+}$in (3.6), we conclude

$$
\sum_{i=1}^{n} \int_{0}^{A} \int_{0}^{T} \int_{\Omega}\left[u_{i}^{*} z_{i}+\left(v_{i}-u_{i}^{*}\right)\left(p_{i}^{u^{*}}-2 u_{i}^{*}\right)\right] \mathrm{d} x \mathrm{~d} t \mathrm{~d} a \leq 0 .
$$


Multiplying (3.5) by $z_{i}$, then integrating over $Q$, we deduce that

$$
\begin{aligned}
& \sum_{i=1}^{n} \int_{0}^{A} \int_{0}^{T} \int_{\Omega}\left(z_{i} u_{i}^{*}\right)(a, t, x) \mathrm{d} x \mathrm{~d} t \mathrm{~d} a \\
& =\sum_{i=1}^{n} \int_{0}^{A} \int_{0}^{T} \int_{\Omega} q_{i}\left(v_{i}-u_{i}^{*}\right) p_{i}^{u^{*}}(a, t, x) \mathrm{d} x \mathrm{~d} t \mathrm{~d} a .
\end{aligned}
$$

Combining (3.7) with (3.8), we have

$$
\sum_{i=1}^{n} \int_{0}^{A} \int_{0}^{T} \int_{\Omega}\left(v_{i}-u_{i}^{*}\right)\left[\left(1+q_{i}\right) p_{i}^{u^{*}}-2 u_{i}^{*}\right] \mathrm{d} x \mathrm{~d} t \mathrm{~d} a \leq 0 .
$$

This completes the proof.

\section{The existence and uniqueness of optimal control}

In this section, we prove the existence and uniqueness of optimal control. From [21], we give the following lemma.

Lemma 4.1 Let $X$ be a reflexive Banach space and let $\varphi: X \rightarrow(-\infty,+\infty$ ] be a lower semicontinuous convex function. If $X_{0}$ is a closed, convex, and bounded subset of $X$, then $\varphi$ attains its infimum on $X_{0}$. In other words, then there is $x_{0} \in X_{0}$ such that

$$
\varphi\left(x_{0}\right)=\inf \left\{\varphi(x), x \in X_{0}\right\} .
$$

The main conclusion is presented as follows:

Theorem 4.1 If $T$ is small enough, then Problem (1.1) has a unique optimal control in $\mathcal{U}$.

Proof For any $u=\left(u_{1}, u_{2}, \ldots, u_{n}\right), v=\left(v_{1}, v_{2}, \ldots, v_{n}\right), u_{i} \in \mathcal{U}_{i}, v_{i} \in \mathcal{U}_{i}, i=1,2, \ldots, n$, we define

$$
H(\varepsilon)=J(\varepsilon u+(1-\varepsilon) v)
$$

we shall prove that $H^{\prime}(\varepsilon)$ is strictly monotone. Indeed, denote by $\left(p_{1}^{\varepsilon}, p_{2}^{\varepsilon}, \ldots, p_{n}^{\varepsilon}\right)$ and $\left(p_{1}^{\varepsilon+\delta}, p_{2}^{\varepsilon+\delta}, \ldots, p_{n}^{\varepsilon+\delta}\right)$ the state corresponding to controls $\varepsilon\left(u_{1}, u_{2}, \ldots, u_{n}\right)+(1-\varepsilon)\left(v_{1}, v_{2}\right.$, $\left.\ldots, v_{n}\right)$ and $(\varepsilon+\delta)\left(u_{1}, u_{2}, \ldots, u_{n}\right)+(1-(\varepsilon+\delta))\left(v_{1}, v_{2}, \ldots, v_{n}\right)$, respectively.

By (1.3), we have

$$
\begin{aligned}
H^{\prime}(\varepsilon)= & \sum_{i=1}^{n} \lim _{\delta \rightarrow 0} \frac{1}{\delta}\left[J\left((\varepsilon+\delta) u_{i}+(1-\varepsilon-\delta) v_{i}\right)-J\left(\varepsilon u_{i}+(1-\varepsilon) v_{i}\right)\right] \\
= & \lim _{\delta \rightarrow 0} \sum_{i=1}^{n} \frac{1}{\delta} \int_{Q}\left[\left(p_{i}^{\varepsilon+\delta}-p_{i}^{\varepsilon}\right)\left(v_{i}+(\varepsilon+\delta)\left(u_{i}-v_{i}\right)\right)+p_{i}^{\varepsilon} \delta\left(u_{i}-v_{i}\right)\right] \mathrm{d} a \mathrm{~d} t \mathrm{~d} x \\
& -\lim _{\delta \rightarrow 0} \sum_{i=1}^{n} \frac{1}{\delta} \int_{Q}\left[\delta^{2}\left(u_{i}-v_{i}\right)^{2}+2\left(v_{i}+\varepsilon\left(u_{i}-v_{i}\right)\right) \delta\left(u_{i}-v_{i}\right)\right] \mathrm{d} a \mathrm{~d} t \mathrm{~d} x \\
= & \sum_{i=1}^{n} \int_{Q}\left[z_{i}^{\varepsilon}\left(v_{i}+\varepsilon\left(u_{i}-v_{i}\right)\right)+p_{i}^{\varepsilon}\left(u_{i}-v_{i}\right)\right] \mathrm{d} a \mathrm{~d} t \mathrm{~d} x \\
& -\sum_{i=1}^{n} \int_{Q} 2\left(v_{i}+\varepsilon\left(u_{i}-v_{i}\right)\left(u_{i}-v_{i}\right)\right) \mathrm{d} a \mathrm{~d} t \mathrm{~d} x,
\end{aligned}
$$


where $z^{\varepsilon}=\left(z_{1}^{\varepsilon}, z_{2}^{\varepsilon}, \ldots, z_{n}^{\varepsilon}\right)$ is the solution of Problem (3.1) corresponding to the control $v_{i}+$ $\varepsilon\left(u_{i}-v_{i}\right)$. By the same argument as given in (3.8), we have

$$
\int_{Q} z_{i}^{\varepsilon}\left(v_{i}+\varepsilon\left(u_{i}-v_{i}\right)\right) \mathrm{d} a \mathrm{~d} t \mathrm{~d} x=\int_{Q} p_{i}^{\varepsilon} q_{i}^{\varepsilon}\left(u_{i}-v_{i}\right) \mathrm{d} a \mathrm{~d} t \mathrm{~d} x .
$$

Combining (4.1) and (4.2), we obtain

$$
H^{\prime}(\varepsilon)=\sum_{i=1}^{n} \int_{Q}\left(u_{i}-v_{i}\right)\left[p_{i}^{\varepsilon}\left(1+q_{i}^{\varepsilon}\right)-2\left(v_{i}+\varepsilon\left(u_{i}-v_{i}\right)\right)\right] \mathrm{d} t \mathrm{~d} t \mathrm{~d} x
$$

where $\left(q_{1}^{\varepsilon}, q_{2}^{\varepsilon}\right)$ is the solution of Problem (3.5) corresponding to the control $v_{1}+\varepsilon\left(u_{1}-v_{1}\right)$ $\left(v_{2}+\varepsilon\left(u_{2}-v_{2}\right)\right)$.

Given $\varepsilon, \rho \in(0,1)$, and $\varepsilon \neq \rho$, and the norm $\|\cdot\|_{L^{2}(Q)}$ being equivalent to the norm $\|\cdot\|_{*}$, combining Theorem 2.1 and Lemma 3.1, we have

$$
\begin{aligned}
& {\left[H^{\prime}(\varepsilon)-H^{\prime}(\rho)\right](\varepsilon-\rho)} \\
& =(\varepsilon-\rho) \sum_{i=1}^{n} \int_{Q}\left(u_{i}-v_{i}\right)\left[p_{i}^{\varepsilon}\left(1+q_{i}^{\varepsilon}\right)-p_{i}^{\rho}\left(1+q_{i}^{\rho}\right)\right] \mathrm{d} a \mathrm{~d} t \mathrm{~d} x \\
& \quad-2(\varepsilon-\rho)^{2} \sum_{i=1}^{n} \int_{Q}\left(u_{i}-v_{i}\right)^{2} \mathrm{~d} a \mathrm{~d} t \mathrm{~d} x \\
& =(\varepsilon-\rho) \sum_{i=1}^{n} \int_{Q}\left[\left(u_{i}-v_{i}\right)\left(p_{i}^{\varepsilon}-p_{i}^{\rho}\right)+\left(u_{i}-v_{i}\right)\left(p_{i}^{\varepsilon} q_{i}^{\varepsilon}-p_{i}^{\rho} q_{i}^{\rho}\right)\right] \mathrm{d} a \mathrm{~d} t \mathrm{~d} x \\
& \quad-2(\varepsilon-\rho)^{2} \sum_{i=1}^{n} \int_{Q}\left(u_{i}-v_{i}\right)^{2} \mathrm{~d} a \mathrm{~d} t \mathrm{~d} x \\
& \leq(\varepsilon-\rho)^{2} \sqrt{K T} \sum_{i=1}^{n}\left\|u_{i}-v_{i}\right\|_{L^{2}(Q)}^{2}+(\varepsilon-\rho)^{2} M_{2} \sqrt{K T} \sum_{i=1}^{n}\left\|u_{i}-v_{i}\right\|_{L^{2}(Q)}^{2} \\
& \quad+(\varepsilon-\rho)^{2} M_{1} \sqrt{\bar{K} T} \sum_{i=1}^{n}\left\|u_{i}-v_{i}\right\|_{L^{2}(Q)}^{2}-2(\varepsilon-p)^{2} \sum_{i=1}^{n}\left\|u_{i}-v_{i}\right\|_{L^{2}(Q)}^{2},
\end{aligned}
$$

where $T$ is small enough. If $\left\|u_{i}-v_{i}\right\|_{L^{2}(Q)} \neq 0$, then we have

$$
\left[H^{\prime}(\varepsilon)-H^{\prime}(\rho)\right](\varepsilon-\rho) \leq-(\varepsilon-\rho)^{2}\left\|u_{i}-v_{i}\right\|_{L^{2}(Q)}^{2}<0 .
$$

Hence, $H^{\prime}(\varepsilon)$ is strictly monotone. Consequently, $J(u)$ is strictly concave in $\mathcal{U}$.

Define the function $\Phi: L^{2}\left(Q ; R^{N}\right) \rightarrow(-\infty,+\infty)$ :

$$
\Phi(u)= \begin{cases}J\left(u_{1}, u_{2}, \ldots, u_{n}\right) & \text { if } u=\left(u_{1}, u_{2}, \ldots, u_{n}\right) \in \mathcal{U}, \\ -\infty & \text { if } u=\left(u_{1}, u_{2}, \ldots, u_{n}\right) \notin \mathcal{U} .\end{cases}
$$

It is clear that $\Phi$ is concave in $L^{2}\left(Q ; R^{N}\right)$. It follows that the nonlinear function $\Phi$ : $L^{2}\left(Q ; R^{N}\right) \rightarrow(-\infty,+\infty)$ is upper weakly semi-continuous. Since $\mathcal{U}$ is a closed, convex, and bounded subset in $L^{2}\left(Q ; R^{N}\right)$ and $J(u)$ is strictly convex in $\mathcal{U}$, by Lemma 4.1, $J(u)$ at- 
tains the unique maximum in $\mathcal{U}$, which implies Problem (1.1) has a unique optimal control in $\mathcal{U}$.

\section{Competing interests}

The authors declare that they have no competing interests.

\section{Authors' contributions}

All authors contributed equally to the manuscript and approved the final version.

\section{Author details}

${ }^{1}$ Institute of Mathematics, Jilin Normal University, Siping, 136000, P.R. China. ${ }^{2}$ Institute of Computer, Jilin Normal University, Siping, 136000, P.R. China.

\section{Acknowledgements}

The authors would like to thank the anonymous referees for their valuable comments on and suggestions regarding the original manuscript. The project is supported by Graduate Student Innovation of Jilin Normal University (201114) and the Department of Education of Jilin Province (2013445).

Received: 23 January 2014 Accepted: 28 May 2014 Published online: 23 September 2014

\section{References}

1. Brokate, M: Pontryagin's principle for control problem in age-dependent population dynamics. J. Math. Biol. 23 75-101 (1985)

2. Murphy, LF, Smith, SJ: Maximum sustainable yield of a nonlinear population model with continuous age structure. Math. Biosci. 104, 259-270 (1991)

3. Murphy, LF, Smith, SJ: Optimal harvesting of age-structured population. J. Math. Biol. 29, 77-90 (1990)

4. Anita, S, lannelli, M, Kim, MY, Park, EJ: Optimal harvesting for periodic age-dependent population dynamics. SIAM J. Appl. Math. 58, 1648-1666 (1998)

5. Anita, S: Optimal harvesting for a nonlinear age-dependent population dynamics. J. Math. Anal. Appl. 226, 6-22 (1998)

6. Ainseba, B, Anita, S, Langlais, M: On the optimal harvesting of a nonlinear age-structured population dynamics. Abstr. Appl. Anal. 6, 357-368 (2001)

7. Busoni, G, Matucci, S: A problem of optimal harvesting policy in two-stage age-dependent populations. Math. Biosci. 143, 1-33 (1997)

8. Luo, Z, Wang, M: Optimal harvesting control problem for linear age-dependent population dynamics. Appl. Math. J. Chin. Univ. Ser. B 18, 412-420 (2003)

9. Gurtin, ME, Murphy, LF: On the optimal harvesting of age-structured populations: some simple models. Math. Biosci. 55, 115-136 (1981)

10. Gurtin, ME, Murphy, LF: On the optimal harvesting of persistent age-structured populations. J. Math. Biol. 13, 131-148 (1981)

11. Liu, Y, Cheng, XL, He, ZR: On the optimal harvesting of size-structured population dynamics. Appl. Math. J. Chin. Univ. 28(2), 173-186 (2013)

12. Zhao, C, Wang, M, Zhao, P: Optimal harvesting problems for age-dependent interacting species with diffusion. Appl. Math. Comput. 163, 117-129(2005)

13. Luo, Z: Optimal harvesting problem for an age-dependent $n$-dimensional food chain diffusion model. Appl. Math Comput. 186, 1742-1752 (2007)

14. Luo, Z: Optimal harvesting control problem for an age-dependent competing system of $n$ species. Appl. Math. Comput. 183, 119-127 (2006)

15. He, ZR: Optimal harvesting of two competing species with age dependence. Nonlinear Anal., Real World Appl. 7 769-788 (2006)

16. He, ZR, Liu, R, Liu, LL: Optimal harvesting for populations system with age-dependent predator. Adv. Math. 42(5), $691-700(2012)$

17. Luo, Z, Xing, T, Li, X: Optimal birth control of free horizon problems for predator-prey system with age-structure. J. Appl. Math. Comput. 34, 19-37 (2010)

18. He, ZR, Liu, LL, Luo, ZX: Global positive periodic solutions of age-dependent competing systems. Appl. Math. J. Chin. Univ. 26(1), 38-46 (2011)

19. Luo, Z, Fan, X: Optimal control for an age-dependent competitive species model in a polluted environment. J. Appl. Math. Comput. 228, 91-101 (2014)

20. Luo, ZX, Yu, XD, Ba, ZG: Overtaking optimal control problem for an age-dependent competition system of $n$ species. Appl. Math. Comput. 218, 8561-8569 (2013)

21. Anita, S: Analysis and Control of Age-Dependent Population Dynamics. Kluwer Academic, Dordrecht (2000)

doi:10.1186/s13661-014-0145-z

Cite this article as: Fu et al.: Optimal harvesting control for an age-dependent competing population with diffusion. Boundary Value Problems 2014 2014:145. 